\title{
EchoGéo
}

$17 \mid 2011$

Activités extractives

\section{Notices bibliographiques}

Notices bibliographiques issues de la Bibliographie Géographique Internationale (base FRANCIS) pour la requête « activités extractives, pétrole, mines, or, fer, gemmes, pays émergents, Afrique ».

\section{Q OpenEdition}

\section{Journals}

Édition électronique

URL : https://journals.openedition.org/echogeo/12571

DOI : 10.4000/echogeo.12571

ISSN : 1963-1197

\section{Éditeur}

Pôle de recherche pour l'organisation et la diffusion de l'information géographique (CNRS UMR 8586)

Référence électronique

« Notices bibliographiques », EchoGéo [En ligne], 17 | 2011, mis en ligne le 27 septembre 2011, consulté le 10 août 2021. URL : http://journals.openedition.org/echogeo/12571 ; DOI : https://doi.org/ 10.4000/echogeo. 12571

Ce document a été généré automatiquement le 10 août 2021.

EchoGéo est mis à disposition selon les termes de la licence Creative Commons Attribution - Pas d'Utilisation Commerciale - Pas de Modification 4.0 International (CC BY-NC-ND) 


\section{Notices bibliographiques}

Notices bibliographiques issues de la Bibliographie Géographique

Internationale (base FRANCIS) pour la requête " activités extractives, pétrole, mines, or, fer, gemmes, pays émergents, Afrique ».

\section{NOTE DE L'ÉDITEUR}

Ces notices bibliographiques sont publiées avec l'autorisation de l'INIST.

1 Légende des zones des références bibliographiques : FT : Titre en français ; ET : Titre en anglais; ST : Titre en espagnol; GT: Titre en allemand; OT : Titre en italien; AU : Auteur; DT: Type de document (Thèse, Congrès); SO: Source; FA: Résumé en français ; EA : Résumé en anglais ; FD : Mots-clés français.

2 FT : L'énergie, l'UE et la Russie

AU : NIES (S.)

DT : Publication en série; Niveau analytique

SO : Hérodote (Paris) ; ISSN 0338-487X ; France ; Da. 2010 ; No. 138 ; Pp. 79-93 ;Bibl. 16 ref. ; 1 tabl., 2 cartes.

FA : Les débats médiatiques se focalisent sur la question de savoir si la Russie, humiliée dans les années 1990, de retour comme puissance énergétique, utilise déjà ou utilisera dans l'avenir l'arme énergétique dans sa relation avec ses clients. La crise gazière russo-ukrainienne de 2009 a démontré la dépendance et la vulnérabilité des uns et des autres, de l'Europe centrale aux Balkans. Les avis sont partagés sur les intentions de la Russie. On peut se demander si l'UE devrait s'alarmer et diversifier ses sources d'approvisionnement. Examen de la relation énergétique UE-Russie dans son contexte historique.

FD: Energie; Russie; Union européenne; Approvisionnement; Dépendance énergétique ; Infrastructure ; Pétrole ; Gaz naturel ; Europe

FT : De la réforme du secteur minier à celle de l'État

AU : MAZALTO (M.)

DT : Publication en série; Niveau analytique

SO : Cahiers africains ; France ; Da. 2009 ; No. 76 ; Pp. 171-189 ; Bibl. 39 ref. 
FA : Analyse des effets de la loi minière de 2002 qui veut assainir un secteur qui fonctionne sur une base informelle contrôlée par des réseaux mafieux. Conflits potentiels entre d'une part les firmes (internationales) auxquelles le gouvernement a accordé de vastes concessions et d'autre part les mineurs artisanaux et les autorités coutumières. Depuis 2008, une commission gouvernementale doit revoir les contrats accordés entre 1996 et 2006.

FD : Mine ; Législation ; Industrie extractive ; Investissement étranger ; Congo

FT : Rentes, territoires et développement : "que tout change pour que rien ne change"

AU : MAGRIN (G.) ; POURTIER (R.), ed.

DT : Publication en série ; Niveau analytique

SO : Bulletin de l'Association de géographes français ; ISSN 0004-5322 ; Coden BAGFAO ; France ; Da. 2010 ; vol. 87 ; No. 1 ; Pp. 56-68 ; Bibl. 35 ref. ; 1 carte.

FA : Tirées de l'exportation de matières premières brutes vers le marché mondial, les rentes n'ont pas jusqu'ici été porteuses de développement, faute d'être investies en faveur des activités productives. Les systèmes rentiers ont contribué à la construction des territoires des jeunes Etats-nations, mais ils s'accompagnent de fragilités économiques et sociopolitiques défavorables au développement. Les concurrences mondiales pour les ressources naturelles risquent de renforcer les travers des systèmes rentiers alors que de nouvelles régulations internationales essaient de réconcilier rentes et développement. Si les logiques de fragmentation et de déconnexion entre ressources et territoires semblent dominer, le nouveau contexte offre aux systèmes rentiers des espaces de bifurcation qui méritent d'être mieux connus.

FD : Rente ; Développement ; Afrique ; Culture de rente ; Mine ; Pétrole ; Territoire

5 FT : (Conflits géo-économiques dans le secteur des combustibles et de l'énergie dans la région de la Caspienne)

OT : Geoèkonomoceskie konflikty v toplivno-ènergeticeskoj sfere Kaspijskogo regiona

AU : KRAMARENKO (A.V.)

DT : Publication en série ; Niveau analytique

SO : Vestnik Moskovskogo universiteta. Seriâ 5: Geografiâ; ISSN 0579-9414; Russie; Da. 2010; No. 1; Pp. 64-71; Bibl. 12 ref.; 1 fig., 2 tabl.

FA: Signification géoéconomique de la région de la Caspienne. Investissements étrangers dans la production de matières premières. Conflits géopolitiques autour de l'infrastructure de transport du pétrole et du gaz.

FD : Conflit ; Géopolitique ; Géo-économie ; Energie ; Combustible ; Pipe-line ; Pétrole ; Gaz naturel ; Russie d'Asie ; Caspienne ; Astrahan

ET: Cruciform sovereignty, matrix governance and the scramble for Africa's oil: insights from chad and Sudan

AU: CARMODY (P.)

DT : Publication en série ; Niveau analytique

SO : Political geography; ISSN 0962-6298; Royaume-Uni ; Da. 2009 ; vol. 28 ; No. 6 ; Pp. 353-361 ; Bibl. 2 p. ; 1 fig.

FA: L'article essaie d'éclairer la manière dont la mondialisation a reconfiguré la souveraineté. Les concepts de gouvernance matricielle et de souveraineté cruciforme permettent un engagement avec le défi de Slater, à savoir théoriser le développement de façon à donner une plus grande priorité à la géopolitique. 
FD : Tchad ; Soudan ; Souveraineté ; Conflit ; Pétrole ; Pipe-line ; Gestion des ressources ; Mondialisation; Niveau de vie

7 ET: India's energy security: challenges and opportunities

AU: DADWAL (S.R.)

DT: Publication en série; Niveau analytique

SO: Eurasian geography and economics; ISSN 1538-7216; Etats-Unis; Da. 2009; vol. 50; No. 6; Pp. 665-681; Bibl. 64 ref.; 5 fig.

FA : Défi de l'énergie en Inde. Stratégie pour les hydrocarbures en Inde : exploration et développement domestiques accélérés; augmentation des parts étrangères; importations; pipelines de gaz naturel et de pétrole; exportations de produits pétroliers; réserves stratégiques. Politique d'énergies renouvelables.

FD : Energie ; Sécurité ; Pétrole ; Gaz naturel ; Charbon ; Energie renouvelable ; Energie nucléaire ; Raffinage ; Réchauffement climatique ; Inde

\section{ET: China's energy security: challenges and priorities}

AU: THOMSON (E.); HORII (N.)

DT: Publication en série; Niveau analytique

SO: Eurasian geography and economics; ISSN 1538-7216; Etats-Unis; Da. 2009; vol. 50; No. 6; Pp. 643-664; Bibl. 82 ref.; 7 fig., 2 tabl.

FA : Revue des sources d'énergie primaire de la Chine : pétrole, charbon, gaz naturel, énergie hydraulique. Stratégies pour renforcer la sécurité énergétique : pétrole, gaz naturel, énergie nucléaire, énergies renouvelables. Amélioration de l'efficacité énergétique.

FD : Energie ; Sécurité ; Pétrole ; Gaz naturel ; Charbon ; Energie renouvelable ; Energie nucléaire ; Réserves énergétiques ; Biocarburant ; Pipe-line ; Chine

$9 \quad$ FT : Pillage et vandalisme dans le Delta du Niger

$\mathrm{AU}$ : AUGÉ (B.)

DT : Publication en série ; Niveau analytique

SO : Hérodote (Paris) ; ISSN 0338-487X ; France ; Da. 2009 ; No. 134 ; Pp. 151-175 ; Bibl. 13 ref. ; 1 carte.

FA : Les attaques de pirates de plus en plus organisés se sont multipliées et ont causé les plus importantes pertes de pétrole de l'histoire du pays. Le Nigéria tente de les juguler. Les militants qui réclament une meilleure répartition des revenus du pétrole n'ont pas besoin de beaucoup développer leur discours pour convaincre la population de la mauvaise gestion du secteur énergétique par l'Etat fédéral. Il doit avoir recours à du pétrole raffiné à l'étranger et les centrales thermiques au gaz subissent des ruptures $\mathrm{d}$ 'approvisionnement alors que le pays possède les premières réserves d'Afrique.

FD : Pillage ; Pétrole; Piraterie ; Violence ; Politique ; Ethnie ; Insécurité ; Energie ; Gestion des ressources; Nigéria ; Conflit

\section{FT : Le rôle des ressources naturelles dans les conflits armés africains}

AU : HUGON (P.)

DT : Publication en série ; Niveau analytique

SO : Hérodote (Paris) ; ISSN 0338-487X ; France ; Da. 2009 ; No. 134 ; Pp. 63-79; Bibl. 38 ref. ; 1 fig., 3 tabl.

FA : Les relations entre guerres et ressources naturelles ont conduit à une écologie politique de la guerre : guerres de ressources, guerres environnementales, de pillage ou de sécession liées aux ressources naturelles. Les ressources du sous-sol peuvent attiser des contrôles par la violence. Les ressources du sol peuvent aviver les tensions. L'auteur 
différencie l'enchevêtrement des facteurs et des acteurs du conflit, l'économie politique des conflits, le rôle spécifique des ressources naturelles.

FD : Guerre; Conflit; Ressource naturelle; Violence; Economie politique; Analyse économique ; Criminalité ; Stratégie d'acteurs ; Minerai ; Afrique

11 FT : Ressources minérales, armes et violences dans les Kivus (RDC)

$\mathrm{AU}$ : JACQUEMOT (P.)

DT : Publication en série ; Niveau analytique

SO : Hérodote (Paris) ; ISSN 0338-487X ; France ; Da. 2009 ; No. 134 ; Pp. 38-62 ; Bibl. 30

ref. ; 2 tabl., 2 cartes

FA : Le lien causal direct entre le commerce des minerais et les conflits persistants dans

ces deux provinces est avéré depuis la publication de plusieurs rapports internationaux. L'exploitation et le trafic du coltan, de l'étain et de l'or, contrôlés par les groupes militaires et des intermédiaires, nourrissent des achats d'armes et entretiennent les tensions. Les répercussions ont été tragiques de 1994 à 2008: déplacements forcés des populations, violences contre les femmes, enrôlement des enfants, bouleversement des équilibres sociaux, pertes écologiques

FD : Ressource naturelle ; Conflit ; Minerai ; Economie souterraine ; Armée ; Commerce ; Crise sociale ; Dégradation de l'environnement ; Congo RD ; Kivu ; Violence

ET: Potentials for migration and mobility among oil workers in the Russian North

AU: SPIES (M.)

DT: Publication en série; Niveau analytique

SO: Geografiska annaler. Series B. Human geography; ISSN 0435-3684; Suède; Da. 2009; vol. 91; No. 3; Pp. 257-273; Bibl. 64 ref.; 10 fig., 1 tabl.

FA : Une mobilité intense caractérise la population du nord de la Russie post-soviétique. Si les périphéries russes sont en déclin, on rencontre aussi des régions et des secteurs industriels prospères, souvent en liaison avec l'exploitation des ressources naturelles. Examen du comportement de mobilité des travailleurs du pétrole. Exemple de la compagnie SeverTEK sise dans la République des Komis, région qui profite de la transition et qui devrait continuer à se développer. La plupart des migrants proviennent de Sibérie, d'Extrême-Orient, des pays nouvellement indépendants de la CEI. Le comportement de mobilité fréquente de l'emploi s'associe à des migrations alternantes sur de grandes distances, que l'on n'utilise pas comme une mesure de décentralisation. On enregistre de fortes probabilités pour des migrations futures. La région peut être quittée si la situation de l'emploi le permet.

FD : Russie du Nord ; Région périphérique ; Mobilité de l'emploi ; Migration; Industrie pétrolière; Restructuration économique; Gestion des ressources; Komi; Russie d'Europe

ET: The other side of civil society story: women, oil and the Niger Delta environmental struggle in Nigeria

AU: ANUGWOM (E.E.); ANUGWOM (K.N.)

DT : Publication en série ; Niveau analytique

SO : GeoJournal ; ISSN 0343-2521 ; Coden GEOJDQ ; Allemagne ; Da. 2009 ; vol. 74 ; No. 4 ; Pp. 333-346 ; Bibl. 34 ref.

FA : En dépit de leur apparente invisibilité dans le discours dominant du delta du Niger, les femmes forment la première ligne de défense sociale contre le dénuement socioéconomique dans la région. Les femmes jouent un rôle actif pour soutenir le développement dans un environnement hostile. Elles contribuent par leur vie 
associative à la consolidation de certains groupes socio-économiques.

FD : Environnement; Développement; Pétrole; Femme; Association; Pauvreté; Géographie sociale; Société civile; Nigéria; Dégradation de l'environnement; Structure socio-économique ; Niger delta

\section{ET: Geopolitics of pipelines and Eastern Europe with especial regard to Hungary} AU: KOCSIS (K.); TINER (T.)

DT: Livre; Niveau analytique

SO: Hungarian Geographical Bulletin; Hongrie; Da. 2009; vol. 58; No. 1; Pp. 49-67; Bibl. 22 ref.; 5 fig., 5 tabl.

EA: The energy strategy of East Central European countries have joined to EU in 2004 is differentiated. It can be stated that majority of these countries have already energy policy and strategy to secure their own energy supply. All of them are making fluent efforts to be independent from considerable part of the Russian oil and gas import in the near or farer future. To avoid negative effects of the futures unforeseen gas wars and unfriendly actions originated from Russia or Ukraine, the new member states of the European Union have worked out more scenarios and projects for the future. The article analyses the geopolitical characteristics of Europe's energy supply and strategy and describes the different pipeline projects.

FD : Energie ; Pipe-line ; Géopolitique ; Pétrole ; Gaz naturel ; Europe de l'Est ; Russie ; Ukraine ; Hongrie

FT : (Territoire, identité et pouvoir en Sierra Leone, du commerce des esclaves aux diamants rouges)

OT : Territorio, identità e potere in Sierra Leone, dallo Slave Trade ai Blood Diamonds AU : PISTOCCHI (F.)

DT : Publication en série; Niveau analytique

SO : Rivista geografica italiana; ISSN 0035-6697 ; Coden RGGIAD ; Italie ; Da. 2009 ; vol. 116 ; No. 1 ; Pp. $47-82$; Bibl. 54 ref. ; 7 fig., 5 phot.

FA : La Sierra Leone a été mêlée à la traite négrière par les Anglais, qui ont fondé après une colonie pour les esclaves affranchis et ont ouvert le Fourah Bay College, université la plus importante de l'Afrique occidentale. Climat, végétation et ressources souterraines (diamants) font appeler le pays la "Suisse de l'Afrique". Malheureusement, le pays est surtout connu par la longue guerre civile meurtrière de 1991-99. Ses origines ne dépendent pas seulement de causes ethniques. Ses aspects historiques, économiques et politiques montrent son niveau de globalisation

FD : Guerre ; Conflit ; Esclavage ; Pouvoir ; Ethnie ; Diamant ; Sierra Leone ; Géographie régionale ; Ressource naturelle; Histoire

FT : Boom aurifère et dynamiques économiques entre Sénégal, Mali et Guinée

AU : MBODJ (F.B.) ; MAGRIN (G.), dir. publ.

DT : Publication en série; Niveau analytique

SO : EchoGéo ; ISSN 1963-1197 ; France ; Da. 2009 ; No. 8 ; Pp. 1-15 ; Bibl. 10 ref. ; 1 fig. coul., 1 tabl., 6 phot. coul., 4 cartes coul.

$\mathrm{FA}$ : La conjoncture mondiale et les réformes minières entreprises par des pays comme la Guinée, le Mali et le Sénégal ont créé les conditions favorables à l'investissement de multinationales de l'or dans un espace transfrontalier situé entre l'est du Sénégal, l'ouest du Mali et le nord-est de la Guinée. A travers les activités annexes et les investissements directs occasionnés, ces multinationales apparaissent comme un facteur d'intégration nationale et sous-régionale de ces zones aurifères jusqu'ici en 
marge. Toutefois, l'insuffisance de leurs contributions financières, la gestion peu efficace de celles-ci, ainsi que la concurrence directe et indirecte que subissent les économies agricoles soulèvent des interrogations sur l'avenir économique des régions étudiées face aux activités extractives.

FD : Mine ; Or ; Investissement ; Entreprise multinationale ; Sénégal ; Mali ; Guinée ; Espace transfrontalier ; Frontière ; Intégration régionale ; Afrique

FT : Le Sénégal Oriental à l'aube du développement minier. Les collectivités locales des jeux du pouvoir aux enjeux du territoire

AU : GREIG (I.) ; MAGRIN (G.), dir. publ.

DT : Publication en série ; Niveau analytique

SO : EchoGéo ; ISSN 1963-1197 ; France ; Da. 2009 ; No. 8 ; Pp. 1-13 ; Bibl. 16 ref. ; 3 cartes coul.

FA : On assiste aux prémices d'un développement minier faisant intervenir des acteurs multiples et inégaux. Les difficultés rencontrées sur le terrain traduisent enjeux invisibles et tensions palpables. Il s'agit d'analyser la position fragile des collectivités locales dans un jeu d'acteurs complexe, placé sous le double signe de la mondialisation économique et d'une décentralisation inaboutie. Région et communautés rurales ont un rôle déterminant à jouer dans l'orientation des dynamiques territoriales, à l'articulation d'un horizon national trop lointain et d'un échelon ultra-local survalorisé.

FD : Stratégie d'acteurs ; Collectivité locale ; Territoire ; Pouvoir ; Exploitation minière ; Développement ; Stratégie de développement ; Sénégal ; Mine ; Sénégal de l'Est

FT : Mine d'or et développement durable. Quelques réflexions sur le site de Sabodala (Sénégal oriental)

AU : LAMINE DIALLO (M.) ; MAGRIN (G.), dir. publ.

DT : Publication en série; Niveau analytique

SO : EchoGéo ; ISSN 1963-1197 ; France ; Da. 2009 ; No. 8 ; Pp. 1-14 ; Bibl. 34 ref. ; 1 phot. coul., 3 cartes coul.

FA : La relance des activités extractives au Sénégal oriental a commencé avec le projet minier de Sabodala. A l'image de sa région (Tambacounda), ce village est une localité enclavée, caractérisée par la domination des activités traditionnelles (agriculture, orpaillage). La pauvreté y est perceptible à travers le manque d'emplois des jeunes, la rareté des infrastructures socio-économiques et des voies de communications. Ainsi, l'installation d'une industrie minière moderne suscite beaucoup d'espoirs auprès des populations villageoises, d'autant plus que l'État et la société adjudicataire du projet placent cette entreprise sous les auspices des principes du développement durable. Mais entre les intentions affichées et les réalités du terrain, l'écart est grand: à l'échelle locale, la mine produit des effets très ambivalents, difficiles à interpréter via les grilles d'analyse du développement durable.

FD : Mine; Or ; Village; Industrie extractive; Développement durable; Désenclavement ; Projet ; Sénégal

\section{ET: China's economic and political penetration in Africa}

AU: PANNELL (C.W.)

DT : Publication en série; Niveau analytique

SO : Eurasian geography and economics ; ISSN 1538-7216 ; Etats-Unis ; Da. 2008 ; vol. 49 ; No. 6 ; Pp. 706-730 ; Bibl. 47 ref. ; 11 fig., 1 tabl.

FA : Les objectifs de la stratégie africaine de la Chine. Le contexte historique récent de 
la politique de la Chine en Afrique. Les buts économiques de la Chine et la stratégie en Afrique. L'Afrique du Sud, l'Egypte, le Soudan et l'Angola.

FD: Stratégie d'acteurs; Politique; Economie; Pétrole; Exportation; Minorité; Chinois; Ressource minière; Commerce international; Chine; Afrique; Afrique du Sud ; Egypte ; Soudan ; Angola

ET: The effects of gas flaring on crops in the Niger Delta, Nigeria

AU: DUNG (E.J.); BOMBOM (L.S.); AGUSOMU (T.D.)

DT : Publication en série; Niveau analytique

SO : GeoJournal ; ISSN 0343-2521 ; Coden GEOJDQ ; Allemagne ; Da. 2008 ; vol. 73 ; No. 4 ; Pp. 297-305 ; Bibl. 31 ref. ; 6 fig., 1 tabl.

EA: This study explores the effects of gas flaring on commonly cultivated crops in the Niger Delta region of Nigeria. It attempts a spatial gradient analysis of crop growth and development near a gas flaring site at Eleme, Rivers state, Nigeria

FD: Agriculture; Pollution; Rendement agricole; Analyse spatiale; Gaz naturel; Feu; Prospection pétrolière; Analyse statistique; Température du sol; Température de l'air; Delta; Nigéria; Niger

21 ET: A risky business: mining, rent and the neoliberalization of "risk"

AU: EMEL (J.); HUBER (M.T.)

DT : Publication en série; Niveau analytique

SO : Geoforum ; ISSN 0016-7185 ; Royaume-Uni ; Da. 2008 ; vol. 39 ; No. 3 ; Pp. 1393-1407 ; Abs. anglais ; Bibl. 100 ref. ; 2 fig.

FA : Les auteurs. étudient comment le concept de "risque" a été investi pour légitimer certains accords biaisés pour répartir la rente entre le capital des sociétés minières et des États propriétaires. Exemple de la liaison entre la promotion d'une politique minière par la Banque Mondiale et la politique tanzanienne à la fin des années 1990 pour attirer les IDE vers la production aurifère.

FD: Mine; Or; Risque; Rente foncière; Néolibéralisme; Exploitation minière; Investissement ; Stratégie de développement ; Tanzanie

FT : (Les potentiels de réhabilitation dans la mine de cuivre de Nkana, Kitwe, Zambie)

GT : Rehabilitationspotenziale in der Nkana-Kupfermine, Kitwe, Zambia

AU : KNIPPERTZ (M.) ; LEHMKUHL (F.)

DT : Publication en série ; Niveau analytique

SO : Geo-öko ; ISSN 1616-0983 ; Allemagne ; Da. 2006 ; vol. 27 ; No. 1-2 ; Pp. 35-51 ; Bibl. 22 ref. ; 16 fig.

FA: La réhabilitation des surfaces extractives dégradées gagne de plus en plus d'importance dans le contexte de gestion et de ménagement des ressources et des matières premières. L'exemple de la mine de Nkana (Zambie) présente les impacts environnementaux et le concept de réhabilitation adapté au lieu.

FD : Zambie ; Kitwe ; Nkana ; Mine ; Réhabilitation; Industrie extractive ; Concept ; Dégradation de l'environnement ; Sol

$23 \quad$ FT : L'Afrique sub-saharienne face aux famines énergétiques

AU : MAGRIN (G.)

DT : Publication en série ; Niveau analytique

SO : EchoGéo ; ISSN 1963-1197 ; France ; Da. 2007-12 ; No. 3 ; 15 p. ; Abs. français/ anglais ; Bibl. 13 ref. ; 2 cartes, 6 phot.

FA: La faible consommation d'énergie est une cause et un symptôme du sous- 
développement de l'Afrique. La pénurie énergétique qui menace le monde semble y introduire deux trajectoires différentes: pour les pays pétroliers, il s'agit d'éviter les pièges de la "malédiction des matières premières", d'utiliser la rente pour consolider l'Etat et l'économie. Pour les autres, il convient déjà de penser à l'après pétrole. Partout, des innovations sont à rechercher pour que l'énergie ne soit plus un frein mais un catalyseur du développement

FD : Energie; Consommation d'énergie; Sous-développement; Stratégie de développement ; Pétrole ; Energie renouvelable ; Afrique

\section{ET: "Fair trade gold": antecedents, prospects and challenges}

AU: HILSON (G.)

DT : Publication en série ; Niveau analytique

SO : Geoforum ; ISSN 0016-7185 ; Royaume-Uni ; Da. 2008 ; vol. 39 ; No. 1 ; Pp. 386-400 ; Abs. anglais; Bibl. 2 p.

FA: Examen critique des défis et impacts liés à l'adoption de modèles d'échanges équitables de produits agricoles dans le secteur artisanal des mines d'or du Ghana. Existence d'un mouvement de protestation en faveur d'un commerce équitable de l'or, qui est lié à l'interaction accrue entre les artisans de la mine, qui recherchent une amélioration de leurs conditions de vie, et les joailliers occidentaux.

FD : Commerce équitable ; Or ; Mine ; Artisanat ; Conditions de vie ; Discours ; Gestion ; Ghana

ET: Contaminates identities: mercury and marginalization in Ghana's artisanal mining sector

AU: TSCHAKERT (P.); SINGHA (K.)

DT : Publication en série; Niveau analytique

SO : Geoforum ; ISSN 0016-7185 ; Royaume-Uni ; Da. 2007 ; vol. 38 ; No. 6 ; Pp. 1304-1321 ; Abs. anglais ; Bibl. 86 ref. ; 5 fig., 2 tabl.

FA: Il s'agit d'un contre-discours par rapport à l'idée de marginalisation et de criminalisation relative à l'extraction illégale de l'or au Ghana. Problèmes complexes, à facettes multiples et conflictuelles liés à l'exploitation minière à petite échelle. Deux sites sont étudiés, et une évaluation de la santé des hommes et de l'environnement est effectuée (pollution par le mercure). Proposition d'une approche d'écologie politique.

FD : Mercure; Marginalité; Secteur informel; Mine; Ecologie politique; Environnement ; Santé ; Pollution ; Or ; Ghana ; Ghana du Sud

ET: The "greening" of global project financing: the case of the Sakhalin-II offshore oil and gas project

AU: BRADSHAW (M.)

DT : Publication en série ; Niveau analytique

SO : Canadian geographer; ISSN 0008-3658; Canada; Da. 2007; vol.51; No. 3 ; Pp. 255-279 ; Abs. anglais/français ; Bibl. 80 ref. ; 4 fig., 1 tabl.

FA: Etude de l'exploitation des réserves de pétrole et de gaz au large de l'île de Sakhaline. Caractère complexe des régions-ressources périphériques. Examen du réseau transnational de défense mis en place pour protester contre le projet énergétique. Enjeux et réponses apportées. Actions récentes menées par le gouvernement russe dans le dossier des impacts environnementaux du projet.

FD : Projet; Energie; Pétrole; Gaz naturel; Financement; Exploitation offshore; Stratégie d'acteurs; Etude d'impact ; Région périphérique; Gestion des ressources; Russie d'Asie ; Dal'nij Vostok; Sahalin ; Ohotsk mer 
ET: Distance between home and workplace as a factor for job satisfaction in the North-West Russian oil industry

AU: SPIES (M.)

DT : Publication en série; Niveau analytique

SO : Fennia ; ISSN 0015-0010 ; Finlande ; Da. 2006 ; vol. 184 ; No. 2 ; Pp. 133-149 ; Abs. anglais ; Bibl. 49 ref. ; 2 fig., 5 tabl.

FA : Après une discussion sur les concepts de migration alternante sur de longues distances et sur les aspects théoriques de la satisfaction procurée par le travail, l'auteur met l'accent sur la situation des travailleurs de l'industrie pétrolière russe. Enquête effectuée dans la république des Komis et le district autonome des Nénets. Facteurs qui influencent la perception des distances. L'allongement croissant des distances ne cause pas nécessairement une satisfaction inférieure. Importance de l'organisation de ces migrations pour une meilleure compréhension des besoins et des stratégies.

FD : Industrie pétrolière; Distance parcourue; Migration alternante; Migration de longue distance ; Perception ; Emploi ; Satisfaction ; Russie d'Europe ; Komi ; Nénets

ET: Russia's oil industry: risk aversion in a risk-prone environment

AU: KRYUKOV (V.); MOE (A.)

DT: Publication en série; Niveau analytique

SO: Eurasian geography and economics; ISSN 1538-7216; Etats-Unis; Da. 2007; vol. 48; No. 3; Pp. 341-357; Abs. anglais; Bibl. 40 ref;; 8 fig., 1 tabl.

FA: Les réserves. Des ressources aux réserves en production. Les stratégies de l'industrie pétrolière : l'investissement pour l'exploration et l'investissement pour la production. Politique d'investissement en amont : perspective d'une entreprise

FD: Industrie pétrolière; Industrie; Gestion du risque; Réserves énergétiques; Exploration ; Investissement ; Production ; Russie

FT : Les filières "pierres précieuses" et "diamant" : la fin de deux trajectoires parallèles

AU : CANAVÉSIO (R.)

DT : Publication en série; Niveau analytique

SO : Cahiers d'Outre-Mer; ISSN 0373-5834; France; Da. 2006; vol.59; No. 236; Pp. 451-462 ; Abs. français/anglais ; Bibl. 13 ref. ; 4 fig., 1 phot.

FA : Les 2 marchés des filières "diamant" et "pierres précieuses" ont longtemps eu des trajectoires parallèles. L'émergence de pays nouveaux producteurs a mis fin à ces trajectoires. La venue du Canada a transformé le circuit de commercialisation des brillants et provoqué l'arrêt progressif de la production des petits pays africains. La découverte des saphirs à Madagascar n'a rien modifié dans le marché des pierres précieuses car l'Etat est incapable de peser sur le système économique mondialisé.

FD : Diamant ; Pierre précieuse ; Exploitation minière ; Commercialisation ; Marché ; Matière première ; Monde ; Afrique du Sud ; Russie ; Canada ; Australie

\section{FT : La Chine à la conquête des marchés énergétiques mondiaux}

AU : MEIDAN (M.)

DT : Publication en série; Niveau analytique

SO : Hérodote (Paris); ISSN 0338-487X ; France; Da. 2007 ; No. 125 ; Pp. 77-94 ; Abs. français/anglais ; Bibl. 42 ref. ; 2 fig.

FA : La nature ambiguë du lien entre gouvernement et compagnies pétrolières chinoises exacerbe les tensions internationales et laisse entendre une politique dirigée par les plus hautes instances du pays pour le partage des ressources mondiales par ses 
compagnies. La stratégie pétrolière internationale est présentée comme un équilibre d'intérêts et de pressions commerciaux et diplomatiques. Il s'agit d'une politique souple et évolutive facilitant la poursuite des objectifs commerciaux tout en modérant leurs effets potentiellement nuisibles.

FD : Energie; Politique énergétique; Consommation d'énergie; Pétrole; Sécurité énergétique ; Marché ; Chine

ET: Strained relations: a critical analysis of the mining conflict in Prestea, Ghana AU: HILSON (G.); YAKOVLENA (N.)

DT : Publication en série ; Niveau analytique

SO : Political geography; ISSN 0962-6298; Royaume-Uni ; Da. 2007 ; vol. 26 ; No. 1 ; Pp. 98-119; Abs. anglais ; Bibl. 68 ref. ; 1 fig., 1 tabl.

FA : Etude de la dynamique d'un conflit opposant des groupes de mineurs indigènes opérant illégalement à une multinationale gérant les ressources minières. Les efforts du gouvernement pour réinstaller les mineurs et leur fournir des projets alternatifs de vie ne sont guère encourageants. Le conflit est survenu car le programme de réformes $d u$ secteur minier, qui accorde la priorité à des projets à grande échelle contrôlés par l'étranger a négligé de se préoccuper suffisamment des moyens d'existence des indigènes.

FD : Or ; Mine ; Conflit ; Conditions de vie ; Régime foncier ; Communauté ; Gestion des ressources ; Entreprise multinationale ; Ghana ; Ghana du Sud-Ouest

\section{ET: Observations on the geographical dimensions of Russia's resource abundance}

AU : BRADSHAW (M.)

DT : Publication en série; Niveau analytique

SO : Eurasian geography and economics ; ISSN 1538-7216 ; Etats-Unis ; Da. 2006 ; vol. 47 ; No. 6 ; Pp. 724-746 ; Abs. anglais ; Bibl. 55 ref. ; 5 fig ; 1 tabl.

FA : La Russie en tant qu'économie de ressources. La géographie des industries de ressources de la Russie. La distribution géographique des prix des ressources en Russie. Les composantes des prix.

FD : Ressource naturelle; Gaz naturel; Pétrole; Métaux; Industrie du bois; Prix; Distribution spatiale ; Développement régional ; Disparités régionales ; Russie

ET: Co-option in Siberia: the case of diamonds and the Vilyuy Sakha

AU: CRATE (S.A.)

DT : Publication en série ; Niveau analytique

SO : Polar geography (1995); ISSN 1088-937X ; Etats-Unis ; Da. 2002; vol. 26 ; No. 4 ; Pp. 289-307 ; Abs. anglais ; Bibl. 37 ref. ; 1 fig.

FA : L'écosystème de Vilyuy, adaptation indigène et colonisation russe. La période soviétique : collectivisation, industrialisation. La période post-soviétique : impacts du barrage hydroélectrique de Vilyuy et de son lac adjacent, contamination chimique des eaux de surface régionales, contamination nucléaire des retombées au cours de la construction $\mathrm{du}$ barrage, efforts officiels pour réconcilier les problèmes environnementaux des régions de Vilnyuy. La naissance et le transfert du comité de Vilyuy. Victoires environnementales dans la Russie post-soviétique et au-delà.

FD : Santé ; Environnement ; Pollution ; Mine ; Diamant ; Post-communisme ; Barrage ; Pollution chimique ; Pollution radioactive ; Russie d'Asie ; Sibir' ; Iakout-Sakha

ET: The regional dimension of Russian oil production: Is a sustained recovery in prospect?

AU : SAGERS (M.J.) 
DT : Publication en série; Niveau analytique

SO : Eurasian geography and economics ; ISSN 1538-7216 ; Etats-Unis ; Da. 2006 ; vol. 47 ; No. 5 ; Pp. 505-545 ; Abs. anglais ; Bibl. 64 ref. ; 8 fig., 7 tabl.

FA : Tendances récentes de la production pétrolière. Prologue : moteurs clés du boom 1999-2004 et du lent déclin 2005-2006. Causes majeures du lent déclin 2005-2006. Contribution de nouveaux champs et de nouveaux projets majeurs. Tendances régionales de production pétrolière: Sibérie occidentale, Sakhalin, Sibérie orientale, Bassin Volga-Oural, bassin Timan-Petchora, champs du Caucase du Nord, oblast de Kaliningrad, mer Caspienne offshore.

FD : Pétrole ; Production ; Région ; Russie ; Sibir' Zapadnaâ ; Sibir' Vostocnaâ ; Sahalin ; Volga ; Ural fleuve ; Kaliningrad ; Caspienne ; Caucase

FT : (Base géographique de la création d'une infrastructure de transport de la province pétrolifère et gazière de Timan-Petchora)

OT: Geograficeskoe obosnovanie sozdaniâ transportnoj infrastruktury TimanoPecorskoj neftegazonosnoj provincii

AU : KOROBOV (V.B.)

DT : Publication en série; Niveau analytique

SO : Izvestiâ Rossijskoj Akademii nauk. Seriâ geograficeskaâ ; ISSN 0373-2444 ; Russie ; Da. 2006 ; No. 4 ; Pp. 87-98 ; Abs. anglais/russe ; Bibl. 19 ref. ; 3 fig., 4 tabl.

FA: Décomposition de systèmes sous forme de hiérarchies. Exemple d'étude d'un système complexe nature-technologie: le transport du gaz et du pétrole de cette province d'Arhangelsk.

FD: Infrastructure; Système complexe; Classification hiérarchique; Transport; Pétrole ; Gaz naturel ; Russie d'Europe ; Arhangel'sk ; Timan-Pecora

FT : Le delta du Niger (Nigeria) : rivalités de pouvoir, revendications territoriales et exploitation pétrolière ou les ferments de la violence

AU : FANCHETTE (S.) ; FANCHETTE (s.), ed.

DT : Publication en série; Niveau analytique

SO : Hérodote (Paris) ; ISSN 0338-487X ; France ; Da. 2006 ; No. 121; Pp. 190-220 ; Abs. français/anglais ; Bibl. 26 ref. ; 2 fig.

FA : Aucun projet de bonification des terres ou d'aménagement hydraulique n'a été mis en place par l'administration territoriale pour limiter l'inondation des terres agricoles et améliorer les conditions de production et de transport. Analyse des modalités de participation des différents acteurs à plusieurs échelles dans le contrôle de la gestion des ressources naturelles et minérales. Facteurs sous-jacents à l'exploitation des ressources naturelles et minières. Raisons du sous-développement critique de cette région, qui subit une dégradation importante de son environnement.

FD : Delta ; Pouvoir ; Pétrole ; Crue ; Politique ; Mise en valeur ; Peuplement ; Ethnie ; Conflit ; Nigéria ; Niger

\section{ET: Gold mining in Ghana's forest reserves: a report on the current debate}

AU: HILSON (G.); NYAME (F.)

DT : Publication en série; Niveau analytique

SO : Area (London); ISSN 0004-0894; Royaume-Uni; Da. 2006; vol. 38 ; No. 2; Pp. 175-185 ; Abs. anglais ; Bibl. 19 ref. ; 1 fig., 2 tabl., 1 phot.

FA : Débat autour de la décision du gouvernement ghanéen d'autoriser l'exploration de l'or et par suite l'exploitation minière dans des réserves forestières protégées. Quatre compagnies minières ont obtenu des concessions dans de telles zones en 2001. Il 
importe de lever les incertitudes sur les implications de ces activités. Les perspectives des indigènes par rapport à ces projets méritent d'être mises à jour, et le dialogue doit être facilité entre les diverses parties prenantes.

FD : Environnement; Exploitation minière; Or; Communauté rurale; Réserves forestières ; Gestion de l'environnement ; Groupe de pression ; Impact ; Mine ; Ghana

FT : Le prix des "arbres sauvages": petite chronique des compensations du Consortium Esso aux paysans tchadiens

AU : SEIGNOBOS (C.) ; MADJIGOTO (R.)

DT : Publication en série; Niveau analytique

SO : Annales de géographie (Paris); ISSN 0003-4010 ; France; Da. 2005 ; vol. 114 ; No. 646 ; Pp. 643-663 ; Abs. français/anglais ; Bibl. 34 ref. ; 2 fig., 1 tabl.

FA : Des compensations ont été versées par le Consortium Esso dans le sud du Tchad pour les terrains occupés et les arbres détruits par le chantier pétrolier. Au-delà des difficultés techniques de leurs évaluations, des rapports de force ont surgi entre le Consortium associé à l'État et les associations locales encadrées par les ONG. Les populations ngambay sont confrontées à une double hypocrisie: celle du développement durable et celle des ONG. Trop éloignées des enjeux de cette exploitation pétrolière, les communautés villageoises ont capté les compensations qui semblaient à leur portée, celle des "arbres sauvages".

FD : Arbre; Essence forestière; Abattage des arbres; Industrie pétrolière; Fruits ; Cueillette ; Foncier ; Compensation financière ; Pétrole ; Village ; Tchad ; Tchad du Sud

FT : La dynamique de l'arrière-pays international des ports camerounais: l'impact du projet pétrolier de Doba sur le port de Kribi

AU : NDJAMBOU (L.E.)

DT : Publication en série; Niveau analytique

SO : Espace géographique (Paris); ISSN 0046-2497; France; Da. 2005 ; vol. 34 ; No. 2 ; Pp. 134-145 ; Abs. français/anglais ; Bibl. 15 ref. ; 3 fig.

FA : Le Tchad et la République centrafricaine, pays enclavés limitrophes du Cameroun, contribuent pour une large part aux performances de ce port. L'auteur montre que dans la perspective de la mise en exploitation des champs pétroliers de Doba au Tchad, le site côtier de Kribi choisi pour abriter le futur terminal pétrolier va favoriser la prééminence du Cameroun dans le traitement des marchandises destinées aux Etats sans littoral de la Communauté économique et monétaire de l'Afrique centrale.

FD : Pétrole; Port ; Arrière-pays ; Pipe-line ; Projet ; Infrastructure ; Impact économique ; Trafic ; Cameroun ; Tchad

FT : (Problèmes géographiques de la mise en valeurs des ressources pétrolières et gazières du Taïmyr)

OT : Geograficeskie problemy osvoeniâ neftegazovyh resursov Tajmyra

AU : RODIN (P.V.)

DT : Publication en série; Niveau analytique

SO : Vestnik Moskovskogo universiteta. Seriâ 5 : Geografiâ ; ISSN 0201-7385 ; Russie ; Da. 2001 ; No. 6 ; Pp. 44-48; Abs. anglais ; 1 fig.

FA: Problèmes d'extraction, de traitement et d'exportation vers d'autres régions. L'auteur envisage et compare deux scenarii de transport de ces hydrocarbures

FD : Pétrole; Gaz naturel ; Mise en valeur; Ressource naturelle; Crise économique ; Scénario ; Transport ; Pipe-line ; Russie d'Asie ; Krasnoârsk ; Taïmyr ; Sibir' Vostocnaâ 


\section{RÉSUMÉS}

Liste des notices bibliographiques obtenues en interrogeant la Bibliographie Géographique Internationale (BGI - bgi-prodig.inist.fr) qui correspond au domaine Géographie de la base FRANCIS (INIST-CNRS, http://www.inist.fr). Pour cette rubrique l'interrogation a été faite sur les années 2005 à 2010 avec la requête « activités extractives, pétrole, mines, or, fer, gemmes, pays émergents, Afrique ».

\section{INDEX}

Thèmes : Sur le Champ - Sur le Terrain 